\title{
Mutations in rugose promote cell type-specific apoptosis in the Drosophila eye
}

\author{
I Wech ${ }^{1}$ and AC Nagel ${ }^{*, 1}$ \\ 1 Institute of Genetics (240), University of Hohenheim, Garbenstr. 30, 70599 \\ Stuttgart, Germany \\ * Corresponding author: AC Nagel, Institute of Genetics (240), University of \\ Hohenheim, Garbenstr. 30, 70599 Stuttgart, Germany. \\ Tel: + 49711 4592210; Fax: + 497114592211 ; \\ E-mail: anjnagel@uni-hohenheim.de
}

Received 30.6.04; revised 12.10.04; accepted 13.10.04

Edited by S Kumar

\begin{abstract}
rugose ( $r g$ ) encodes an A kinase anchor protein and was isolated as a genetic interactor of the Notch and epidermal growth factor receptor (EGFR) pathways during eye development in Drosophila. rg mutants display a small, rough eye phenotype primarily caused by the loss of cone cells. Here we show that the basis of this phenotype is cell type-specific apoptosis rather than transformation and hence can be rescued by reduction of proapoptotic signals. Moreover, a nearly complete rescue is observed by an increased Notch signal suggesting an antiapoptotic function of Notch in this developmental context. Cone cell loss in $r g$ mutants is accompanied by enhanced Jun $\mathrm{N}$-terminal kinase activity and, concomitantly, by a reduction of EGFR signalling activity. Together, these findings support the idea that $r g$ plays an important role in the integration of different signals required for the exact regulation of cone cell development and survival. Cell Death and Differentiation (2005) 12, 145-152.

doi:10.1038/sj.cdd. 4401538
\end{abstract}

Keywords: rugose; anchor protein; cone cell apoptosis; Notch pathway; EGFR pathway; JNK pathway; Drosophila

Abbreviations: DIAPI, Drosophila inhibitor of apoptosis protein I; EGFR, epidermal growth factor receptor; hid, head involution defective; JNK, Jun N-terminal kinase; MAPK, mitogen-activated protein kinase; PCD, programmed cell death; PKA, protein kinase A; rpr, reaper, rg, rugose; SEM, scanning electron microscopy

\section{Introduction}

Programmed cell death (PCD) is an important process for the proper development and homeostasis of multicellular organisms. In Drosophila, the induction of apoptosis requires the activity of the closely linked proapoptotic genes reaper (rpr), head involution defective (hid) and grim, which induce cell death by activating a family of executing proteases known as caspases. ${ }^{1,2}$ Cell death is suppressed by the Drosophila inhibitor of apoptosis protein I (DIAPI), which directly inhibits caspase activity and promotes their ubiquitination and subsequent degradation. ${ }^{3}$ Recent findings suggest that Rprmediated DIAP1 inhibition leads to an induction of Jun Nterminal kinase (JNK) signalling, which was shown to induce apoptosis during imaginal disc development in Drosophila. ${ }^{4-6}$ However, the functional relevance of JNK activation for killing by Reaper is still controversial. ${ }^{7}$

A large number of cells undergo apoptosis during fly embryonic and imaginal development as well as during metamorphosis. One of the best tissues to investigate this process is the eye of the fly, taking into account that it is not an essential structure and easily allows comparative phenotypic analyses. The Drosophila eye is composed of approximately 750 ommatidia, each built up of a fixed number of cells. Centrally located are eight photoreceptor cells, covered by four cone cells and surrounded by two primary pigment cells. ${ }^{8}$ The ommatidia are separated by interommatidial cells, which differentiate into secondary and tertiary pigment cells and interommatidial bristles. Excess cells are eliminated through apoptosis resulting in the precise hexagonal cellular lattice. ${ }^{8,9}$ It was shown that this spatially restricted cell death depends on the balance between epidermal growth factor receptor (EGFR) and Notch receptor signalling. For example, laser ablation-induced cell death during pupal eye development was strikingly blocked by the expression of the activated ras $^{V 12}$ allele, a downstream component of the EGFR/mitogenactivated protein kinase (MAPK) pathway. ${ }^{10}$ Two independent studies revealed that MAPK signalling counteracts apoptosis by downregulating the function and expression of the proapoptotic factor hid. ${ }^{11,12}$ Hence, EGFR signalling supports cell survival, whereas activation of the transmembrane receptor Notch in the interommatidial lattice promotes cell death. ${ }^{9,10,13,14}$

Genetic screens identified rugose $(r g)$ as an interactor of the Notch and EGFR signalling pathway during eye development. ${ }^{15,16} \mathrm{rg}$ encodes a Drosophila A kinase anchor protein. ${ }^{17}$ Mutants in $r g$ are characterized by a rough eye phenotype caused by the loss of cone cells accompanied by further irregularities in the cellular architecture of the pupal retina. ${ }^{15,16,18}$

It was suggested that cone cell loss is based on transformation, albeit the final fate of these cells remained elusive. ${ }^{16}$ In this study, we reinvestigated the basis of the observed cone cell loss in rg mutant eyes. Our analyses indicate that this phenotype is a result of cell type-specific apoptosis rather than cell fate transformation to $1^{\circ}$ pigment cells and occurs largely autonomously in $r g$ mutant cells. Accordingly, the mutant phenotype is completely restored by providing survival signals. Moreover, an increase of Notch signalling rescues cone cell death as well, suggesting that Notch provides an anti- rather than proapoptotic signal in this developmental context. Cone cell loss in $r g$ mutants is accompanied by an increase of JNK activity, whereas MAPK activity is lowered. This study supports the notion that the major role of $r g$ might be the integration of the crosstalk between different signalling pathways to allow precise 
regulation of factors involved in cone cell formation and survival.

\section{Results}

\section{rg mutation leads to the death of cone cells}

$r g$ was isolated as a strong enhancer of the rough eye phenotype caused by the overexpression of the Notch antagonist Hairless $(H)$ in the differentiating eye. ${ }^{15}$ The primary cause of the rough appearance of $\mathrm{rg}$ mutant eyes appears to be a lack of cone cells, which can be visualized by cobalt sulphide stainings of pupal retinae (Figure $\left.1 c^{\prime}\right) .{ }^{15,16}$ In order to follow the fate of the individual cone cells, we used an intermediate allele, $\mathrm{rg}^{\mathrm{E16}}$, that

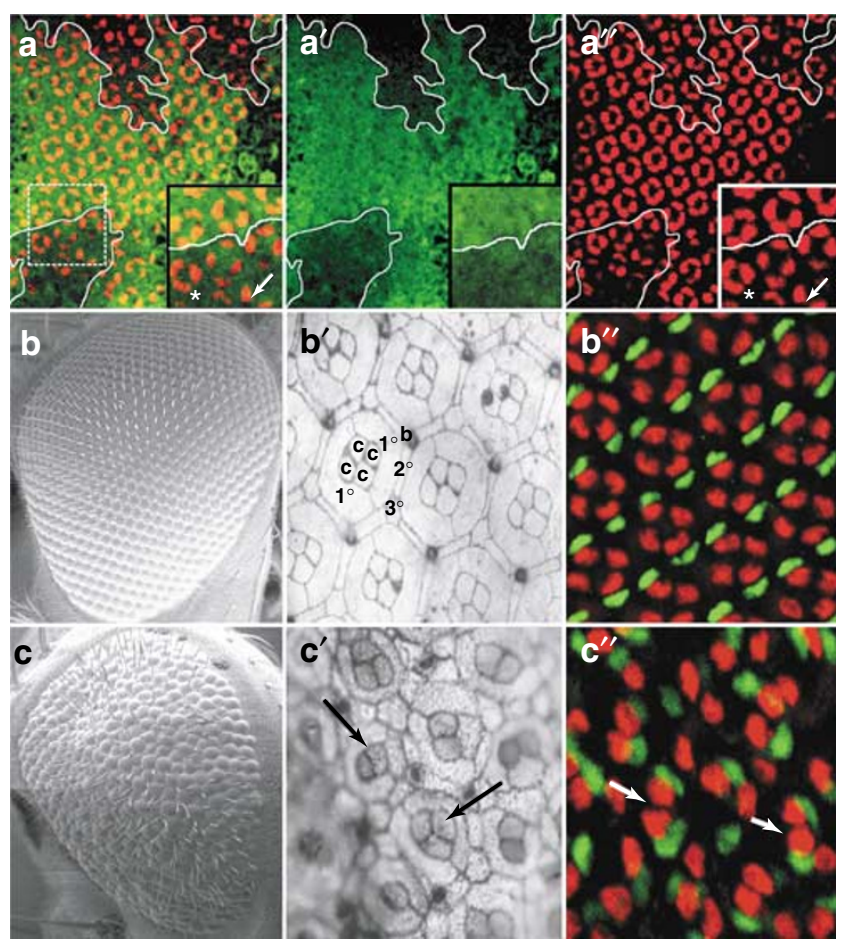

Figure 1 Specific loss of cone cells in $r g^{E 16}$ mutant pupal retinae. (a-a') Pupal eye discs with $\mathrm{rg}^{E 16}$ mutant cell clones are marked by the absence of lacZ, stained with antibodies to $\beta$-galactosidase (green, $\mathbf{a}, \mathbf{a}^{\prime}$ ). Cone cells are visualized with anti-Cut staining (red, $\left.a, a^{\prime \prime}\right)$. The clone borders are outlined. Ommatidia within mutant clones have a reduced number of Cut-positive cone cells. Frequently, only three (asterisk) or even just two (arrow) cone cells remain. Ommatidia close to the clone borders usually have the full complement of four cone cells, implying a positive influence from wild-type cells. Note that Cut levels appear reduced in many mutant cone cell nuclei (see inset). (b) Ommatidia of wild-type eyes are ordered regularly (scanning electron microscopy (SEM) picture). ( $\left.b^{\prime}\right)$ Cobalt sulphide staining of $40-48 \mathrm{~h}$ old pupae reveals the typical arrangement of four cone cells (c) surrounded by two primary pigment cells $\left(1^{\circ}\right)$ and cells of the interommatidial lattice $\left(2^{\circ}, 3^{\circ}\right.$ pigment cells, interommatidial bristle (b)). ( $\left.\mathbf{b}^{\prime \prime}\right)$ Antibody staining against Cut (red) and BarH1 (green) was used as marker for cone cell and $1^{\circ}$ pigment cell fate, respectively. Note the normal arrangement of four cone cells surrounded by two $1^{\circ}$ pigment cells in each ommatidium. (c-c $\left.\mathbf{c}^{\prime \prime}\right) r g^{E 16} / Y$ mutant eyes. Hemizygous $r g^{E 16}$ mutants display a rough eye phenotype (SEM picture (c)), caused by the specific loss of cone cells (arrows in $\mathbf{c}^{\prime}$ ). A loss of Cut-positive cone cells (red) is apparent, whereas the number of BarH1-positive $1^{\circ}$ pigment cells (green) is unchanged ( $c^{\prime \prime}$, white open arrows exemplify ommatidia lacking cone cells) develops a pronounced cone cell loss phenotype little obscured by other retina defects. ${ }^{15}$

Cone cells could be identified because they specifically express Cut, whereas $1^{\circ}$ pigment cells strongly accumulate BarH1 protein. ${ }^{19,20}$ Using specific antibodies directed against these cell markers allowed to distinguish cone cells from $1^{\circ}$ pigment cells in the pupal retinae (Figure 1 $\mathrm{c}^{\prime \prime}$ ). Early recruitment of cone cells seemed undisturbed in $\mathrm{rg}^{E 16}$ since Cut staining was indistinguishable from wild type in the larval eye disc and pupal retina of $24 \mathrm{~h}$ old pupae (not shown). However, during retina maturation, in about $40 \mathrm{~h}$ old pupae ommatidia lacked one to two cone cells on average compared to the four cone cells seen in the wild type. In contrast, $1^{\circ}$ pigment cells seemed little affected (Figure $1 \mathrm{~b}-\mathrm{c}^{\prime \prime}$ ). As $\mathrm{rg}$ encodes a protein kinase $A(P K A)$ anchor protein, one might expect its requirement within the cell of expression. In accordance, $r g$ mutant cell clones revealed cell autonomy of the mutant phenotype (Figure 1a-a'). However, we note that ommatidia close to clone borders showed a full complement of four cone cells more frequently than ommatidia within the clone, suggesting a positive input from surrounding wild-type cells. Moreover, Cut expression appeared fainter in some mutant nuclei suggesting an incomplete transformation into a different cell type or fading away of these cells (Figure $1 \mathrm{a}$ and $\mathrm{a}^{\prime \prime}$ ).

\section{rg mutations result in cell type-specific cell death and not in transformation to $1^{\circ}$ pigment cells}

Lack of cone cells could be a result of transformation into another cell fate. As no systematic increase of $R 7$ cells is observed, a transformation into the previous cell fate seems very unlikely. ${ }^{15,16,21}$ Similarly, the number of $1^{\circ}$ pigment cells, which constitute the subsequent cell fate, is also not increased (Figure $1 c^{\prime \prime}$ ). However, it is conceivable that cone cells are fated for $1^{\circ}$ pigment cells and replace them. The normal $1^{\circ}$ pigment cell might either die as a consequence of wrong signalling input or governed into the next fate, that is, interommatidial lattice cells, eventually forming $2^{\circ}$ or $3^{\circ}$ pigment cells. This is not without precedence. For example, in the sevenless mutant, the presumptive R7 cell is fated for and replaces the normal cone cell. ${ }^{21}$ Since the number of lattice cells is reduced by PCD during final stages of retina maturation, such a transformation would not be detectable by an increase of pigment cell numbers. Alternatively, cone cells could simply die in the $r g^{E 16}$ mutants maybe due to reduction of survival signals.

In order to distinguish between these possibilities, we increased survival signals in two ways. First, we overexpressed the antiapoptotic factor DIAP1 in cone cell precursors and second, we halved the dose of the proapoptotic genes hid, rpr and grim with a Df(3L) $\mathrm{H} 99$ heterozygous background. We not only observed a complete rescue of the adult eye phenotype (Figure 2a and c) but also of the cone cell loss (Figure $2 a^{\prime}$ and $c^{\prime}$ ) without affecting the number of $1^{\circ}$ pigment cells (Figure 2a"). Apparently, the missing cone cell(s) specifically die in the $r g^{E 16}$ mutant. This can be visualized in a double labelling for Cut and activated Caspase-3, which accumulates in the dying cells (Figure 2e). 


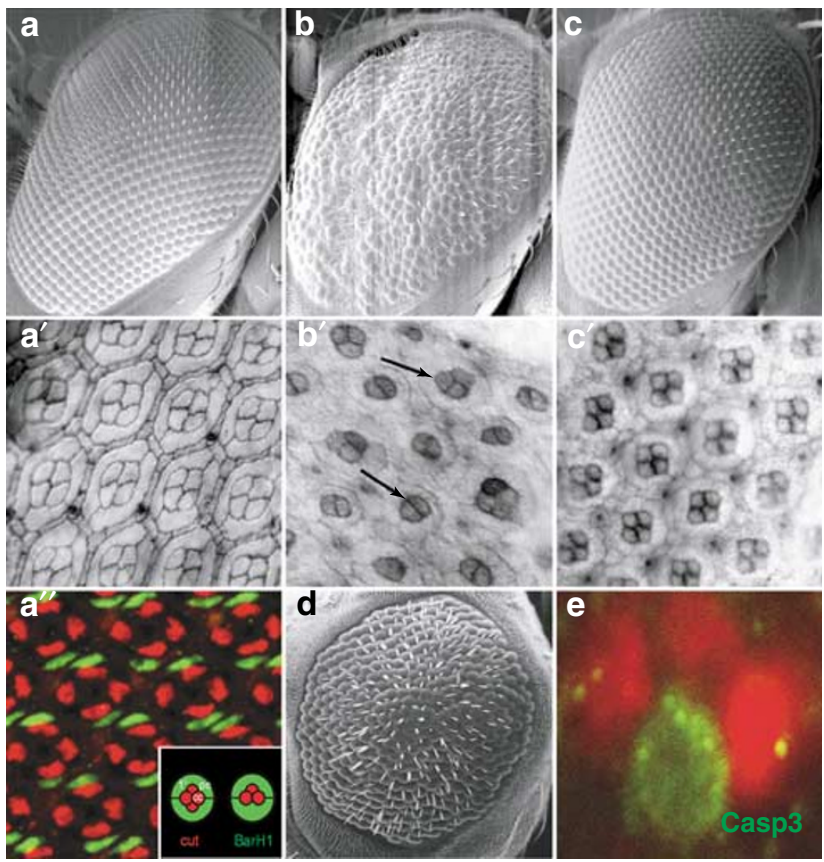

Figure 2 The $r g^{E 16}$ mutant eye phenotype involves cone cell-specific cell death rather than cell type transformation. $\left(\mathbf{a}-\mathbf{a}^{\prime \prime}\right) \mathrm{rg}^{E 16} / \mathrm{Y} ; \mathrm{Df}(3 \mathrm{~L}) \mathrm{H}^{99} /+$ flies. The rough eye phenotype and the specific loss of cone cells in $\mathrm{rg}^{E 16}$ mutants are completely rescued in a heterozygous $D f(3 L) H 99$ background where the gene dose of the proapoptotic genes rpr, hid and grim is reduced by half. The inset in $\left(\mathbf{a}^{\prime \prime}\right)$ represents a schematic drawing of the cellular make-up of a wild-type (left) versus an $r g^{E 16}$ mutant (right) pupal ommatidium where cone cells are marked for Cut protein in red and $1^{\circ}$ pigment cells are marked for BarH1 protein in green. $\mathrm{rg}$ mutants are typified by a reduced number of cone cells but normal $1^{\circ}$ pigment cell numbers. $\left(\mathbf{b}-\mathbf{b}^{\prime}\right) r g^{E 16} / Y ; s e v G a / 4 /+$. The rough appearance of $r g$ mutant eyes is not influenced by the sev-Gal4 driver line (b) nor is the cone cell loss $\left(\mathbf{b}^{\prime}\right)$. $\left(\mathbf{c}-\mathbf{c}^{\prime}\right)$ $\mathrm{rg}^{E 16} \mathrm{Y}$; sevGal4 > UAS DIAP1 flies. Overexpression of the antiapoptotic factor DIAP1 in cone cell precursors results in a complete wild-type appearance of the eye (c) and the survival of all four cone cells despite an $\mathrm{rg}^{E 16} / Y$ mutant background $\left(\mathbf{c}^{\prime}\right)$. Compare the adult eyes from (b) and (c) and the cobalt sulphide staining from $\left(\mathbf{b}^{\prime}\right)$ with $\left(\mathbf{c}^{\prime}\right)$, respectively. The arrow in $\left(\mathbf{b}^{\prime}\right)$ points to examples of ommatidia lacking one or two cone cells. (d) $r g^{E 16} / Y, t h^{5} /+$. Accordingly, the eye phenotype of $r g^{E 96}$ is enhanced when the gene dose of DIAP1 is halved (compare with Figure 1c). (e) $r g^{E 16}$ pupal eye disc (48 h after pupariation) stained with $\alpha$-Cut (red) and $\alpha$-cleaved Caspase-3 (green). Note that one cell in the ommatidium, which by position seems to be a cone cell albeit lacking the Cut marker, undergoes cell death and consequently accumulates activated Caspase-3

Moreover, reducing the activity of the antiapoptotic factor DIAP1 in a $t h^{5}$ heterozygous mutant background, which itself is phenotypically wild type, enhances the rough eye phenotype caused by $\mathrm{rg}^{E 16}$ (Figure 2d, compare with Figure 1c). Protecting cone cells from apoptotic signals in $\mathrm{rg}$ mutants allows them to survive and to form a normal complement of four (Figure $2 \mathrm{a}^{\prime}$ and $\mathrm{c}^{\prime}$ ). Reduction of the proapoptotic gene rpr to one copy in the $X R 38$ mutation results in a slight suppression of the $r g^{E 16}$ rough eye phenotype, suggesting an involvement of rpr in cone cell death (not shown). The fact that the number of $1^{\circ}$ pigment cells is not changed indicates that no cell type transformation occurs (Figure 2a" $\mathrm{a}^{\prime \prime}$ ). Repression of apoptosis by overexpression of DIAPI in cone cells supplies additional survival signals and leads to an inhibition of normal PCD in interommatidial lattice cells also in an rg mutant background (Figure $2 \mathrm{c}^{\prime}$ ). This does, however, not cause any apparent rough eye phenotype in adults (Figure 2c).

\section{Increase in Notch signalling rescues cone cell death in rg mutants}

Genetic interactions between $r g^{E 16}$ and different components of the Notch signalling pathway were already described. ${ }^{15,16}$ It was shown for example that the loss of one copy of the Notch ligand Delta and therefore a reduction in Notch signalling results in a strong enhancement of the rough eye phenotype caused by $r g^{E 16}$. On the other hand, reducing the gene dose of $H$ by half resulted in a rescue of the $r g$ rough eye phenotype. ${ }^{15,16} \mathrm{H}$ acts as antagonist in the Notch signalling pathway by assembling a repressor complex together with $\mathrm{Su}(\mathrm{H})$ and corepressors on Notch target genes. ${ }^{22,23}$ Hence, lowering $\mathrm{H}$ activity should lead to an increase of Notch signalling activity, which might serve as survival signal for cone cells. However, it was shown earlier that Notch promotes PCD in interommatidial lattice cells. ${ }^{10}$ Maybe $H$ and $r g$ mutants interact without involvement of Notch signalling. Indeed, $\mathrm{Su}(\mathrm{H})$ independent activities of $\mathrm{H}$ have been described earlier for aspects of wing and bristle development. ${ }^{24,25}$

This reasoning led us to address whether a reduction of $H$ to one copy also rescues the cone cell death in $\mathrm{rg}^{E 16}$ mutants. In fact, pupal retinae of $\mathrm{rg}^{E 16} ; \mathrm{H}^{P 8} /+$ males were indistinguishable from wild type (Figure $3 a$ and b). Thus, a reduction of $H$ gene activity in an $\mathrm{rg}^{\mathrm{E16}}$ mutant background yields an antiapoptotic signal apparently sufficient to counteract the death stimulus resulting from loss of $r g$ activity, thereby repressing cone cell-specific cell death. Apparently, one role of $\mathrm{H}$ during retina development is to provide a proapoptotic stimulus in accordance with earlier observations that overexpression of $\mathrm{H}$ causes loss of eye or wing tissue. ${ }^{25,26}$ We next asked whether this survival signal resulting from reduced $\mathrm{H}$ activity involves an increase in $\mathrm{N}$ signal. This question is rather difficult to address directly, as overexpression of Notch interferes with multiple aspects of eye development and causes a wide array of phenotypes. ${ }^{9,27,28}$ As predicted, our attempts to rescue $\mathrm{rg}$ mutant phenotype by overexpression of the intracellular domain of $\mathrm{N}$ or of $\mathrm{Su}(\mathrm{H})$ was uninformative due to vast disorganization of the retina (not shown). We therefore doubled the amount of Notch activity in an $\mathrm{rg}^{E 16}$ mutant background by including a second Notch gene copy with the cos 479 transgene. As predicted, the adult rough eye phenotype as well as loss of cone cells was rescued by the extra Notch gene dose (Figure $3 d$ and $d^{\prime}$ ). These results are consistent with the idea that a Notch signal supports cell survival in this context.

\section{EGFR signalling is reduced in $r g$ mutants}

Different genetic screens have revealed numerous interactions between mutants in $r g$ and EGFR signalling components. In general, a reduction of EGFR signalling enhances the $r g$ rough eye phenotype. ${ }^{15,16}$ EGFR has been implicated in the inhibition of proapoptotic signals and the survival of interommatidial lattice cells. ${ }^{10-12}$ Thus, mutants in EGFR signalling components might result in a reduction of survival stimuli, thereby enhancing the defects of $r g$ mutants. In accordance, mutants in the MAPK rolled $\left(r l^{1}\right)$ have rough eyes and lack cone cells as well. ${ }^{29}$ Moreover, heterozygosis for the nuclear effector pointed (pnt $\Delta 88 /+$ ) caused a gentle 

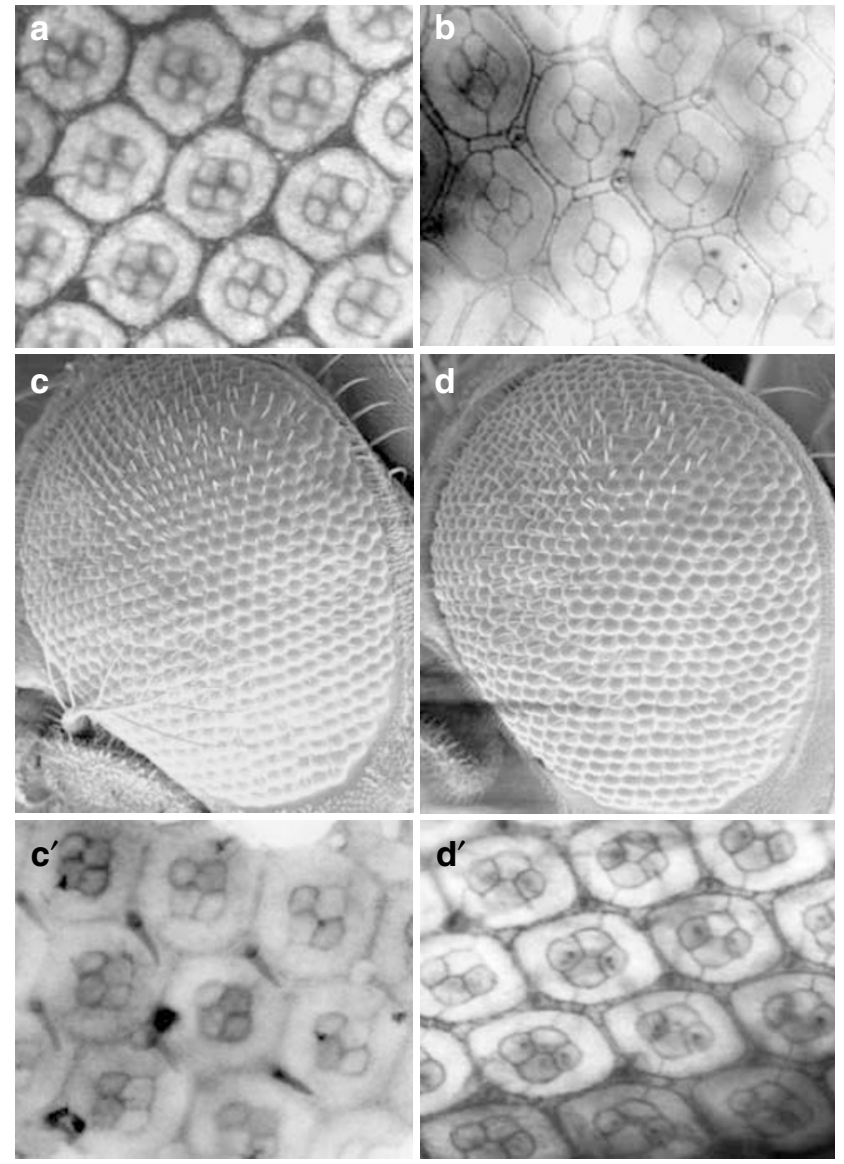

Figure 3 Cell death in $\mathrm{rg}^{E 16}$ eye discs is influenced by Notch pathway components. (a) Pupal eye disc ( $48 \mathrm{~h}$ after pupariation) of $H^{P 8} /+$ reveals a wildtype number of cone cells. (b) The same is seen in $\mathrm{rg}^{E 16} / \mathrm{Y} ; \mathrm{H}^{\mathrm{P}} /+$ double mutants. Thus, cone cell loss of $r g^{E 16}$ mutants is completely rescued by halving the $H$ gene copy. (c- $\mathbf{c}^{\prime}$ ) Adult eyes and pupal eye discs of $N^{t s} / Y$; $\cos 479 / T M 3 S b$ males, which have an additional copy of Notch as transgene, appear superficially quite normal (c) and have a wild-type assembly of cone cells $\left(\mathbf{c}^{\prime}\right)$. $\left(\mathbf{d}-\mathbf{d}^{\prime \prime}\right)$ The $\mathrm{rg}^{E 16}$ eye phenotype is nearly completely rescued by such an additional copy of Notch $\left(r g^{E 16} / Y ; \cos 479 /+\right)$. Only a mild roughening of the adult eye remains (d), whereas the pupal cone cell equipment is completely restored $\left(\mathbf{d}^{\prime}\right)$

enhancement of the $r g^{E 16}$ rough eye phenotype (not shown). Conversely, an increase in EGFR activity should result in a rescue. This is indeed observed: halving the gene dose of the transcriptional repressor anterior open (aop) rescued the $r g^{E 16}$ rough eye phenotype as well as the cone cell loss in pupal retinae to nearly wild type (Figure 4a and a'). As EGFR signal mediates survival of cone cells, does a mutation in $r g$ affect the levels of EGFR signalling? Consistent with this hypothesis, we found a significant decrease of the levels of activated MAPK (diP-ERK) in $r g^{E 16}$ mutant heads to about $24 \%$ of wild-type levels, similar to the levels observed in $S^{E 10}$ heterozygotes (Figure $4 \mathrm{e}$ and $\mathrm{f}$ ). This finding was confirmed by antibody staining against diP-ERK in pupal retinae. In agreement with published data, ${ }^{30,31}$ a strong, punctate signal was detected within wild-type cone cells (Figure $4 b-b^{\prime \prime}$ ). Although this punctate localization of diP-ERK remained unchanged in $r g^{E 16}$ mutants, the intensity of the staining was remarkably lowered (Figure $\left.4 c-c^{\prime \prime}\right)$. These results were confirmed in $r g^{E 16}$ mutant cell clones, where the signalling
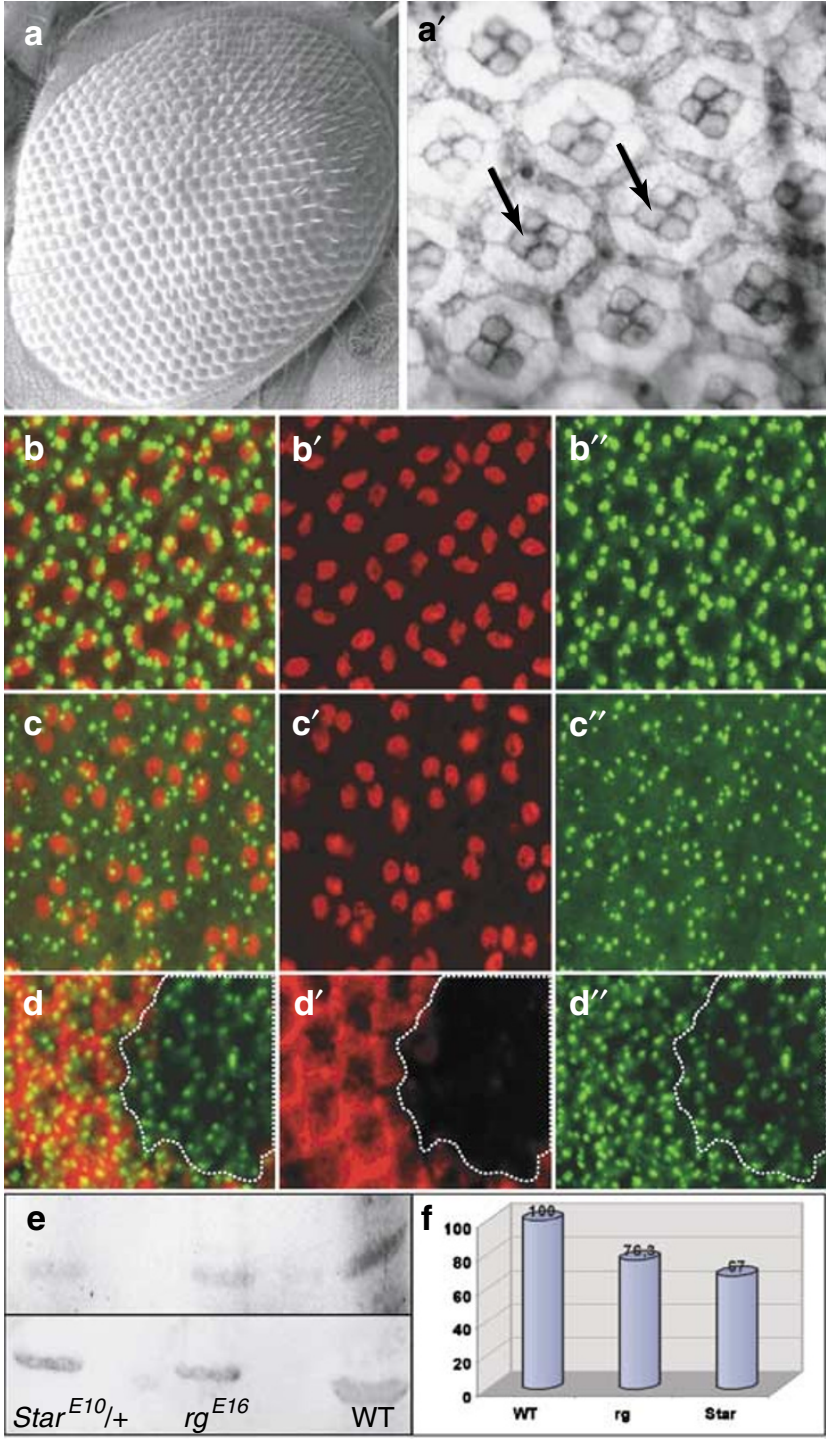

Figure 4 MAPK signalling is reduced in $\mathrm{rg}^{E 16}$ mutant retinae. Halving the gene dose of anterior open (aop), which acts as a repressor of EGFR target gene activation, suppresses the $r g^{E 16}$ rough eye phenotype $\left(\mathbf{a} ; \mathrm{rg}^{E 16} / \mathrm{Y} ; \mathrm{aop} /+\right)$ and restores the four cone cells in pupal retinae ( $\mathbf{a}^{\prime}$, arrows). Pupal retinae ( $48 \mathrm{~h}$ old $)$ from wild-type $\left(\mathbf{b}-\mathbf{b}^{\prime \prime}\right)$ and $r g^{E 16}$ males $\left(\mathbf{c}-\mathbf{c}^{\prime \prime}\right)$ were stained with $\alpha$-Cut (red) and $\alpha$-diPERK (green), respectively. Note the dotted staining representing activated MAKP in wild-type cone cells $\left(\mathbf{b}, \mathbf{b}^{\prime \prime}\right)$, which is likewise present, however, at a reduced level in the $r g^{E 16}$ mutant $\left(\mathbf{c}, \mathbf{c}^{\prime \prime}\right)$, which also reveals the aforementioned reduction of Cut-positive cells (compare $\mathbf{b}-\mathbf{b}^{\prime \prime}$ with $\mathbf{c}-\mathbf{c}^{\prime \prime}$ and $\mathbf{b}^{\prime}$ with $\mathbf{c}^{\prime}$ ). (b) and (c) are merged images. (d-d $\mathbf{d}^{\prime \prime}$ ) Pupal eye disc with an $\mathrm{rg}^{E 16}$ mutant cell clone marked by the absence of lacZ, stained with antibodies to $\beta$-galactosidase (red, $\left.d, d^{\prime}\right)$. The clone border is outlined. The signal intensity of the activated MAPK (green in $\mathbf{d}, \mathbf{d}^{\prime \prime}$ ) in cells within the mutant clone is clearly weaker than in their wildtype neighbours. (e) Western blot analysis of protein extracts from 20 adult heads each detected a less strong signal of the activated MAPK in $r g^{E 16}$ as well as in heterozygous $\mathrm{Star}^{\mathrm{F}}$ mutant heads in comparison to the wild type (upper panel). Lower panel: Loading control $(\alpha$-actin). (f) Signals of three independent experiments were quantified by densitometry and normalized to the values of the loading control. This analysis reveals that the levels of activated MAPK are reduced by approximately $24 \%$ in the $\mathrm{rg}^{\mathrm{E} 16}$ mutants compared to the wild type. In two attempts, protein extracts of $S_{a r} F^{10} \%$ mutant heads were loaded as a positive control showing a reduction of at least $30 \%$ of activated MAPK protein 
activity of activated MAPK within the mutant area was clearly weaker than in the neighbouring wild-type cells (Figure $4 d-$ $\left.d^{\prime \prime}\right)$. Together, these experiments suggest that the PKA anchor protein $\mathrm{Rg}$ is required for normal EGFR signalling activity and thereby may regulate levels of survival signals in the developing retina.

\section{JNK signalling is activated in $\mathbf{r g}$ mutants}

Recently, it was shown that the JNK pathway promotes cell death during the development of imaginal discs. ${ }^{5,6}$ We thus wondered whether the cell death observed in $r g$ mutants is mediated by an increase in JNK signalling. We addressed this question two-fold. First we asked whether reduction in JNK signalling might rescue the $r g$ mutant phenotype. Indeed, lowering the gene dosage of the JNKKKK misshapen ( $m s n)$ or the JNK basket (bsk) by one-half significantly rescued the $\mathrm{rg}^{E 16}$ rough eye phenotype as well as cone cell loss (Figure $\left.5 a-b^{\prime}\right)$. Second, we addressed JNK activity directly by analysing the levels of the activated, that is, phosphorylated protein in wild type versus the rg mutant. Indeed, an increase up to $23 \%$ was observed in $r g^{E 16}$ mutants compared to wildtype heads, whereas in heterozygous $b s k^{1}$, protein levels were reduced by $16 \%$ (Figure $5 f$ and $g$ ). This observation was further confirmed in situ: the activated JNK accumulates in cone cells of the wild-type ${ }^{32}$ and much stronger in the remaining cone cells of $\mathrm{rg}^{E 16}$ mutant retinae as well as in $\mathrm{rg}^{\mathrm{E} 16}$ mutant cell clones when compared to wild-type cells (Figure $5 c-\mathrm{e}^{\prime \prime}$ ). We conclude that cone cell loss in rg mutants is mediated by an enforced signal of the JNK pathway.

\section{Discussion}

In this article, we focused on the analysis of the rough eye phenotype caused by mutations at the $r g$ locus. Mutations in $r g$ interfere with the correct pattern formation of the retina affecting nearly all retinal cell types to some degree, photoreceptor cells, cone and $2^{\circ}$ pigment cells. ${ }^{15,16,18}$ In

Figure 5 JNK pathway signalling is enforced in $\mathrm{rg}^{E 16}$ mutant retinae. Reducing the activity of JNK pathway signalling in a heterozygous $m s n\left(\mathbf{a}^{\left.-\mathbf{a}^{\prime}\right)}\right.$ or $b s k\left(\mathbf{b}-\mathbf{b}^{\prime}\right)$ mutant background clearly suppresses the $\mathrm{rg}^{E 16}$ rough eye phenotype (a, b; compare with Figures $2 \mathrm{c}$ and $3 \mathrm{~b}$ ) and the typical cone cell loss, visualized by cobalt sulphide stainings of the pupal retinae $\left(\mathbf{a}^{\prime}, \mathbf{b}^{\prime}\right.$, arrows). Moreover, programmed cell death of lattice cells in bsk heterozygotes is partly suppressed $\left(\mathbf{b}^{\prime}\right.$, arrowhead). Activated JNK was detected with specific antibodies (JNK-P, green) in $48 \mathrm{~h}$ old wild-type (c-c ${ }^{\prime \prime}$ ) and $r g^{E 16}$ pupae $\left(\mathbf{d}-\mathbf{d}^{\prime \prime}\right)$. In comparison to the wild type, the mutant showed a stronger signal in the cone cells ( $\alpha$-Cut, red) (compare c, $\mathbf{c}^{\prime \prime}$ with $\left.\mathbf{d}, \mathbf{d}^{\prime \prime}\right)$. (c) and (d) are merged images. (e- $\left.\mathbf{e}^{\prime \prime}\right) \mathrm{rg}^{E 16}$ mutant cell clone (clone border is outlined) marked by the absence of lacZ, stained with antibodies against $\beta$-galactosidase (red, e, $\mathbf{e}^{\prime}$ ). On average, we detected a stronger JNK signal within the mutant cone cells (green, $\mathbf{e}, \mathbf{e}^{\prime \prime}$, arrow), which is in line with the results we obtained in the $r g^{E 16}$ mutant retinae (d). The results were quantified by densitometry of Western blots (f). Data were compiled from three independent experiments, and for $b s k^{1}$ from two experiments $(\mathbf{g})$. Protein extracts from 20 heads each (wild type, WT, $r g^{E 16}$ males and $b s k^{1} /+$ flies) were probed for activated JNK using anti-JNK-P antibodies (upper panel) and antiactin antibodies (lower panel) for a loading control. (g) Quantification by densitometry of three independent experiments reveals an enhancement of activated JNK up to $23 \%$ in the $r g^{E 16}$ mutants in comparison to the wild type, whereas in the heterozygous $b s k^{1}$ mutants a reduction of JNK activity of $16 \%$ was observed addition, optic lobe defects were described. ${ }^{18}$ The major defect, however, is a loss of cone cells, which is the primary phenotype even in weaker alleles. ${ }^{15,16}$ Our work followed in finer detail the fate of these cells. Previously, it was suggested that the cone cells fail to differentiate in the mutant. ${ }^{16}$ However, the cone cells, which are born already during late third larval instar, express the Cut marker indistinguishable
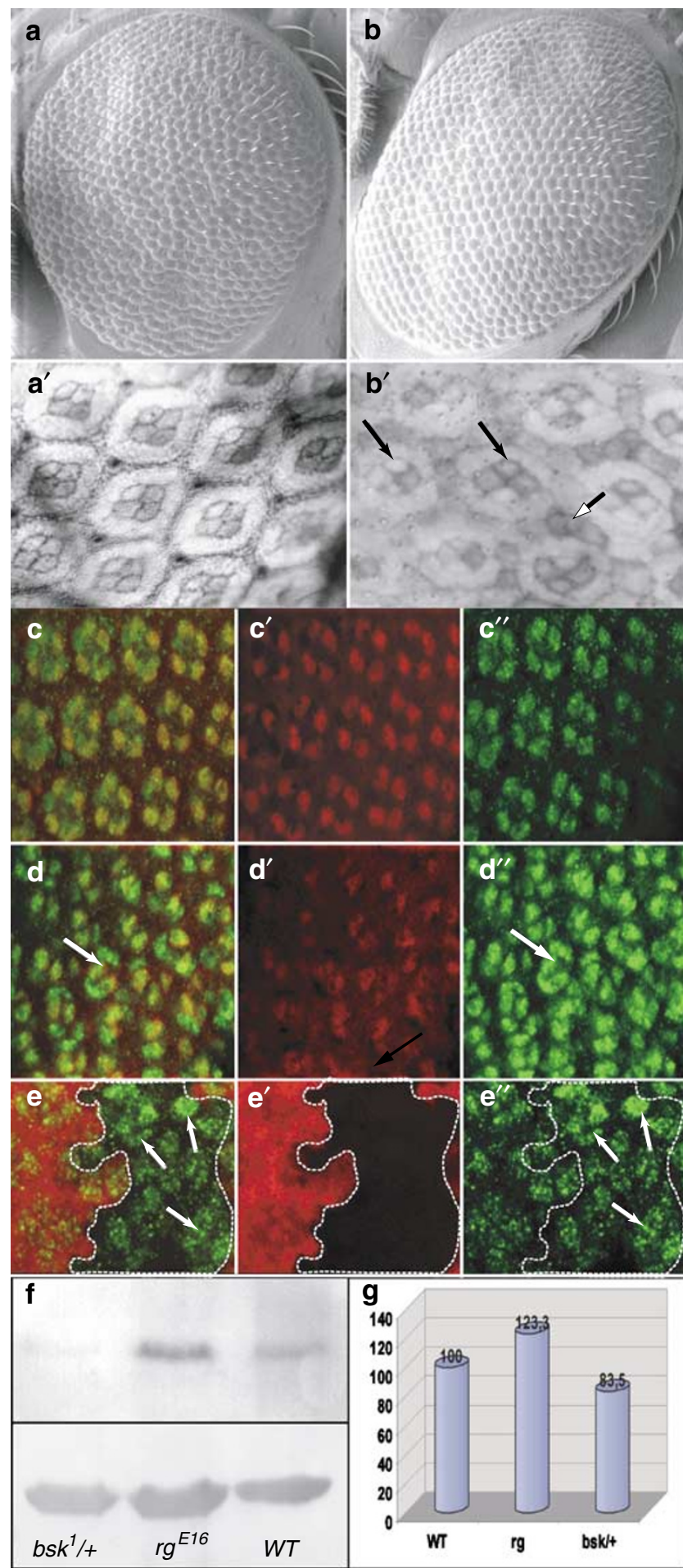
from wild type. This suggests that cone cell recruitment is not disturbed by the rg mutant. Instead, during mid-pupal stages, cone cells are lost by cell type-specific cell death, explaining the failure to detect these cell types in the pupal retinae. ${ }^{16}$ We favour the hypothesis that the AKAP Rg might serve to integrate survival signals during retina development. Presumably, cone cells undergo a specific phase of high sensitivity towards such signals. Thus, a reduction in $r g$ activity might cause sporadic to massive loss primarily of cone cells.

Our findings suggest that activation of the JNK pathway contributes to the apoptotic signal in rg mutant cone cells. In Drosophila, signalling through JNK is required for a variety of processes during embryonic and adult development. ${ }^{33}$ Recently, it was observed that the JNK pathway might also function as a kind of 'developmental supervisor' during wing development. ${ }^{5,6}$ There it was shown that a deregulation of the positional information maintained by Dpp and Wg signalling results in an induction of the JNK pathway followed by an increased level of apoptosis. Thus, the JNK pathway is obviously activated to maintain correct development when normal signalling is distorted. ${ }^{5}$

Cone cell development also depends on multiple inputs, mainly on the multifunctional Notch and EGFR signals and the transcriptional activator lozenge. ${ }^{34,35}$ In $\mathrm{rg}$ mutants, the correct temporal activation level of these signals seems to be impaired, followed by a wrong combinatorial code for cone cell fate development. This could cause increase in JNK signalling and subsequently cone cell-specific cell death. Thus, ablation of cone cells by apoptosis may be a mechanism to proceed with normal retinal development despite impaired normal signalling.

\section{Notch signalling counteracts cone cell death in rg mutants}

Remarkably, an increased Notch signal, either by doubling the gene dose of $N$ or halving the dose of the Notch antagonist Hairless $(H)$, suppresses the rg-specific cone cell death. Notch signalling has previously been associated with both negative and positive effects on apoptosis. A constitutive activated Notch receptor is able to induce cancer and cell transformation probably by impairing apoptosis. ${ }^{36,37}$ Moreover, it has been demonstrated that an activated form of Notch is able to inhibit apoptosis mediated by presenilin in Drosophila, leading to the speculation that an activated Notch signalling might protect from apoptotic cell death. ${ }^{38}$ However, Notch has also been implicated in the induction of apoptosis during different developmental stages in Drosophila, for example, during terminal mitotic divisions of the Drosophila CNS, PNS and wing development. ${ }^{39-41}$ Interestingly, genetic studies revealed that Notch is also able to induce apoptosis during eye development in the pupal retina. ${ }^{9,42}$ There it was shown that Notch signalling within the interommatidial lattice cells, which surround the individual ommatidia, promotes cell death, whereas a signal mediated by the activated EGFR promotes cell survival. ${ }^{10,14}$ In rg mutants, an increase in Notch signalling activity results in effects comparable to those of an enforced EGFR activation, indicating that both pathways might adopt an antiapoptotic function and therefore behave as 'survival signals' in this developmental context.

\section{How can $r g$ influence the activity of the EGFR and other signalling pathways?}

A kinase anchoring proteins (AKAPs) contribute to the spatial and temporal PKA signalling specificity by targeting PKA to specific substrates and subcellular compartments. ${ }^{43}$ rg encodes for one of the two known A kinase anchor proteins in Drosophila. ${ }^{15-17}$ Reducing the activity of the catalytic subunit of Pka in a pka-C1 $1^{01272}$ heterozygous mutant background results in an enhancement of the $r g$ mutant eye phenotype (not shown), supporting the involvement of $r g$ in PKA signalling. ${ }^{44}$ Although AKAPs are characterized on the basis of their interaction with PKA, they also serve as scaffolding proteins by binding other signalling molecules like kinases and phosphatases as well as components of various other signalling pathways. ${ }^{45,46}$ We observe that in $r g$ mutants the signalling through the canonical MAP kinase pathway was clearly decreased. This cascade proceeds from the membrane-bound GTP-binding protein Ras, to the sequential activation of at least three Ser-Thr kinases leading to the specific activation of nuclear target genes. ${ }^{47}$ Reducing the activity of $r g$ might cause mislocalization of activated PKA molecules to the plasma membrane thereby reducing the basal activity of PKA important for the tight regulation of this pathway. Moreover, by interfering with the Ser-Thr kinases itself, rg might be important to ensure that signals pass quickly from one kinase to the next following, thus preventing an interplay with functionally unrelated kinases in the same cell.

Interestingly, in addition to their interaction with signal terminators like kinases or phosphatases, AKAPs also assemble signals derived from multiple pathways into multiprotein signalling complexes organizing responses to external and intrinsic signals in different organisms. ${ }^{45,46,48}$ One might speculate that $r g$ is an important factor for the integration and precise regulation of the different factors known to be involved in cone cell development.

\section{Materials and Methods}

\section{Fly work}

Flies were cultured on standard fly food and maintained at $18^{\circ} \mathrm{C}$. Crosses were performed at $25^{\circ} \mathrm{C}$. Pupae were staged by collecting late third instar larvae and incubating them for $24-48 \mathrm{~h}$ at $25^{\circ} \mathrm{C}$ and $48-96 \mathrm{~h}$ at $18^{\circ} \mathrm{C}$, respectively. The following fly stocks were used in this study: Oregon-R, $b k^{1}$, Df(3L) H99/TM3Sb, $m n^{102}, m^{172}$, pnt488, Star ${ }^{E 10}, t^{5}$ (Bloomington Stock Center), aop (stock center Tübingen), $H^{P 8}$ (provided by D Maier) ${ }^{49}, N^{t s}$; cos 479 (provided by A Preiss), ${ }^{50}$ pka $^{01272}$ (gift from $S$ Cohen), ${ }^{44}$ arm-lacZFRT19A; eyFlp (provided by F Pignoni), FRT19A (provided by $\mathrm{D}$ Ebacher), XR38 (gift of $\mathrm{K}$ White). ${ }^{51}$ Eye-specific overexpression was achieved with the Gal4/UAS system ${ }^{52}$ using sev$\mathrm{Gal}^{53}$ as driver line and UAS DIAP I (gift of Arno Müller).

Clones mutant for $r g^{E 16}$ were induced in presumptive eye tissue by heat shocking $r g^{E 16}$ FRT19A/arm-lacZ FRT19A; ey-Flp/ + third instar larvae for $1 \mathrm{~h}$ at $37^{\circ} \mathrm{C}$ as described by Newsome et al. ${ }^{54}$ Adult eyes were dehydrated in ethanol, covered with a mixture of gold and pallodium and 
analysed with a digital scanning microscope from Zeiss and the Orion version 6 software.

\section{Staining procedures}

Pupal eye discs shown in this paper (40-48 $\mathrm{h}$ after pupal formation ) were dissected in PBS and fixed in 4\% paraformaldehyde for $20 \mathrm{~min}$. After washing four times with PBT (PBS $+0.1 \%$ Tween) and blocking for $1 \mathrm{~h}$ with $4 \%$ normal goat serum, the retinae were incubated overnight at $4{ }^{\circ} \mathrm{C}$ with the respective primary antibodies. After several washes in PBT, respective goat secondaries coupled to DTAF or Cy3 were added ( $1: 200$; Jackson Immuno Research). The following primary antibodies were used: mouse anti-Cut $(1: 20)$, obtained from the Developmental Studies Hybridoma Bank (University of lowa, NICHD contract NO1-HD-7-3262), rabbit anti-BarH1, ${ }^{20}$ rabbit anti-cleaved Caspase-3 (1:200; NEB Cell Signalling Technology), rabbit-anti $\beta$-Gal (1:5000; Rockland, USA), rabbit anti-active MAPK $(1: 100)$ and rabbit anti-active JNK-P $(1: 500$; both Promega). The specificities of the diPERK and JNKP antibodies were controlled with the help of the Gal4/UAS system: overexpression of the dominant-negative form of EGFR (UAS EGFR $\left.{ }^{\mathrm{DN}}\right)^{55}$ using EqGal4 ${ }^{56}$ results in a loss of diPERK staining along the dorso-ventral boundary in pupal retinae, whereas the overexpression of the activated JNKK (UAS hep $\left.{ }^{\text {act }}\right)^{31}$ leads to an increase in JNKP staining.

Pictures were taken with a BioRad MRC 1024 confocal system linked to a Zeiss Axioskop using BioRad Laser Sharp 3.1 software. Cobalt sulphide staining was performed as previously described by Wolff and Ready. ${ }^{8}$

\section{Detection of activated kinases}

In all, 20 heads each of $\mathrm{rg}^{E 16}$ hemizygous males, wild-type OregonR1 males and heterozygous $S t a F^{E 10}$ and $b s k^{1}$ males were homogenized in $100 \mu$ l Em-Lys-buffer (10 mM HEPES pH 7.6, 5 mM EGTA, 5 mM EDTA, $1 \%$ Triton $\mathrm{X}-100,1 \mu \mathrm{l}$ protein inhibitor cocktail (Roche)) and boiled for $5 \mathrm{~min}$. After pelleting the debris, $10 \mu \mathrm{l}$ of each homogenate was separated on $10 \%$ PAGE and blotted on nitrocellulose. Membranes were probed with rabbit anti-active JNK-P (1:500, Promega, recognizes a $44 \mathrm{kDa}$ band), rabbit anti-active MAPK (1:500, Promega, recognizes an $80 \mathrm{kDa}$ band) and rabbit anti-actin $(1: 250$, Sigma, recognizes a band at $42 \mathrm{kDa})$ antibodies as a loading control. The signals were quantified by densitometric scanning of the dry membranes using the wincam software program and normalized with the actin control.

\section{Acknowledgements}

We thank S Cohen, D Ebacher, T Kojima, D Maier, A Müller, S Noselli, L Pignoni, A Preiss and the Bloomington stock center for flies and/or antibodies. We are indebted to W Ulrich for taking the SEM pictures, D Müller for taking pictures of $r g$ eye clones and W Staiber for photographic work. We also thank S Schreiber for the initiation of these studies and T Stösser for technical assistance. We are grateful to A Preiss for critical reading of the manuscript and $A$ Preiss and $D$ Maier for helpful discussions throughout the work. This work was supported by the DFG Grant NA 427/ $1-1$ to $\mathrm{ACN}$.

\section{References}

1. Bangs $P$ and White $K(2000)$ Regulation and execution of apoptosis during Drosophila development. Dev. Dyn. 218: 68-79
2. Vernooy SY, Copeland J, Ghaboosi N, Griffin EE, Yoo SJ and Hay BA (2000) Cell death regulation in Drosophila: conservation of mechanism and unique insights. J. Cell Biol. 150: F69-F76

3. Palaga T and Osborne B (2002) The 3D's of apoptosis: death, degradation and DIAP's. Nat. Cell Biol. 4: E149-E151

4. Kuranaga $\mathrm{E}$, Kanuka $\mathrm{H}$, Igaki T, Sawamoto $\mathrm{K}$, Ichijo $\mathrm{H}$, Okano $\mathrm{H}$ and Miura $\mathrm{M}$ (2002) Reaper-mediated inhibition of DIAP1-induced DTRAF1 degradation results in activation of JNK in Drosophila. Nat. Cell Biol. 4: 705-710

5. Adachi-Yamada T, Fujimura-Kamada K, Nishida Y and Matsumoto K (1999) Distortion of proximodistal information causes JNK-dependent apoptosis in Drosophila wing. Nature 400: 166-169

6. Moreno E, Basler K and Morata G (2002) Evolution of TNF signaling mechanisms: JNK-dependent apoptosis triggered by Eiger, the Drosophila homolog of the TNF superfamily. Curr. Biol. 12: 1263-1268

7. Chen P, Ho SI, Shi Z and Abrams JM (2004) Bifunctional killing activity encoded by conserved reaper proteins. Cell Death Differ. 11: 704-713

8. Wolff T and Ready DF (1991) The beginning of pattern formation in the Drosophila compound eye: the morphogenetic furrow and the second mitotic wave. Development 113: 841-850

9. Cagan RL and Ready DF (1989) Notch is required for successive cell decisions in the developing Drosophila retina. Genes Dev. 3: 1099-1112

10. Miller DT and Cagan RL (1998) Local induction of patterning and programmed cell death in the developing Drosophila retina. Development 125: 2327-2335

11. Kurada P and White K (1998) Ras promotes cell survival in Drosophila by downregulating hid expression. Cell 95: 319-329

12. Bergmann A, Agapite J, McCall K and Steller H (1998) The Drosophila gene hid is a direct molecular target of Ras-dependent survival signaling. Cell 95: 331-341

13. Freeman M (1996) Reiterative use of the EGF receptor triggers differentiation of all cell types in the Drosophila eye. Cell 87: 651-660

14. Sawamoto K, Taguchi A, Hirota Y, Yumada C, Jin M and Okano H (1998) Argos induces programmed cell death in the developing Drosophila eye by inhibition of the Ras pathway. Cell Death Differ. 5: 262-270

15. Schreiber S, Preiss A, Nagel AC, Wech I and Maier D (2002) Genetic screen for modifiers of the rough eye phenotype resulting from overexpression of the Notch antagonist Hairless in Drosophila. Genesis 33: 141-152

16. Shamloula HK, Mbogho MP, Pimentel AC, Chrzanowska-Lightlowlers ZMA, Hyatt V, Okano K and Venkatesh T (2002) rugose (rg), a Drosophila A kinase anchor protein, is required for retinal pattern formation and interacts genetically with multiple signaling pathways. Genetics 161: 693-710

17. Han J-D, Baker NE and Rubin CS (1997) Molecular characterization of a novel A kinase anchor protein from Drosophila melanogaster. J. Biol. Chem. 272: 26611-26619

18. Shamloula HK (2003) Mutation in rugose gene locus affects the pigment cell number and alters the optic lobe phenotype in the Drosophila visual system. J. Egypt. Soc. Parasitol. 33: 955-967

19. Blochlinger K, Bodmer R, Jan LY and Jan YN (1990) Patterns of expression of Cut, a protein required for external sensory organ development in wild-type and cut mutant Drosophila embryos. Genes Dev. 4: 1322-1331

20. Hayashi T, Kojima T and Saigo K (1998) Specification of primary pigment cell and outer photoreceptor fates by BarH1 homeobox gene in the developing Drosophila eye. Dev. Biol. 200: 131-145

21. Tomlinson A, Bowtell DD, Hafen E and Rubin GM (1987) Localization of the sevenless protein, a putative receptor for positional information, in the eye imaginal disc of Drosophila. Cell 51: 143-150

22. Morel V, Lecourtois V, Massiani $O$, Maier $D$, Preiss $A$ and Schweisguth $F$ (2001) Transcriptional repression by Suppressor of Hairless involves the binding of a Hairless-dCTBP complex in Drosophila. Curr. Biol. 11: 1964-1976

23. Barolo S, Stone T, Bang AG and Posakony JW (2002) Default repression and Notch signaling: Hairless acts as an adaptor to recruit the corepressors Groucho and dCtBP to Suppressor of Hairless. Genes Dev. 16: 1964-1976

24. Nagel $A C$, Maier $D$ and Preiss $A(2000) \mathrm{Su}(\mathrm{H})$-independent activity of Hairless during mechano-sensory organ formation in Drosophila. Mech. Dev. 94: 3-12

25. Johannes B and Preiss A (2002) Wing vein formation in Drosophila: Hairless is involved in the cross-talk between Notch and EGF signaling pathways. Mech. Dev. 115: 3-14

26. Go MJ, Eastman DS and Artavanis-Tsakonas S (1998) Cell proliferation control by Notch signaling in Drosophila development. Development 125: 2031-2040 
27. Baker NE (2002) Notch and the patterning of ommatidial founder cells in the developing Drosophila eye. In Drosophila Eye Development Moses K, ed Berlin, Heidelberg: Springer-Verlag pp. 35-54

28. Nagaraj $R$, Canon $J$ and Banerjee $U$ (2002) Cell fate specification in the Drosophila eye. In Drosophila Eye Development Moses K, ed Berlin, Heidelberg: Springer-Verlag pp. 73-84

29. Kumar JP, Hsiung F, Powers MA and Moses K (2003) Nuclear translocation of activated MAP kinase is developmentally regulated in the developing Drosophila eye. Development 130: 3703-3714

30. Lesokhin AM, Yu SY, Katz J and Baker NE (1999) Several levels of EGF receptor signaling during photoreceptor specification in wild-type, Ellipse, and null mutant Drosophila. Dev. Biol. 205: 129-144

31. Weber U, Eroglu C and Mlodzik M (2003) Phospholipid membrane composition affects EGF receptor and Notch signaling through effects on endocytosis during Drosophila development. Dev. Cell 5: 559-570

32. Jarvis BW, Schaefer EM and Miller DT (1997) Detection of JNK, p38 and MAPK enzyme activation using anti-dual phosphopeptide antibodies: coordinated signaling of the extracellular signal-regulated protein kinase (ERK) superfamily. Promega Notes Mag. 63: 2-7

33. Davis RJ (2000) Signal transduction by the JNK group of MAP kinases. Cell 102: 239-252

34. Flores GV, Duan H, Yan H, Nagaraj R, Fu W, Zou Y, Noll M and Banerjee U (2000) Combinatorial signaling in the specification of unique cell fates. Cell 103 75-85

35. Fu W, Duan H, Frei E and Noll M (1998) shaven and sparkling are mutations in separate enhancers of the Drosophila Pax2 homolog. Development 125: 2943-2950

36. Artavanis-Tsakonas S, Rand MD and Lake RJ (1999) Notch signaling: cell fate control and signal integration in development. Science 284: 770-776

37. Mumm JS and Kopan R (2000) Notch signaling: from the outside in. Dev. Biol. 228: 151-165

38. Ye Y and Fortini ME (1999) Apoptotic activities of the wild-type and Alzheimer's disease-related mutant presenilins in Drosophila melanogaster. J. Cell Biol. 146: 1351-1364

39. Lundell MJ, Lee H-K, Perez E and Chadwell $L$ (2003) The regulation of apoptosis by Numb/Notch signaling in the serotonin lineage of Drosophila. Development 130: 4109-4121

40. Orgogozo V, Schweisguth F and Bellaiche $Y$ (2002) Binary cell death decisions regulated by unequal partitioning of Numb at mitosis. Development 129: 4677-4684

41. Milan M, Perez L and Cohen SM (2002) Short-range cell interactions and cell survival in the Drosophila wing. Dev. Cell 2: 797-805
42. Yu S, Yoo SJ, Yang L, Zapata C, Srinivasan A, Hay BA and Baker NE (2002) A pathway of signals regulating effector and initiator caspases in the developing Drosophila eye. Development 129: 3269-3278

43. Scott JD and McCartney S (1994) Localization of A-kinase through anchoring proteins. Mol. Endocrinol. 8: 5-11

44. Kalderon D and Rubin GM (1988) Isolation and characterization of Drosophila cAMP-dependent protein kinase genes. Genes Dev. 2: 1539-1556

45. Feliciello A, Gottesman ME and Avvedimento EV (2001) The biological functions of A-kinase anchor proteins. J. Mol. Biol. 308: 99-114

46. Tasken $\mathrm{K}$ and Aandahl EM (2004) Localized effects of cAMP mediated by distinct routes of protein kinase A. Physiol. Rev. 84: 137-167

47. Casci T and Freeman M (1999) Control of EGF receptor signalling: lessons from fruitflies. Cancer Metast. Rev. 18: 181-201

48. Herrgard S, Jambeck P, Taylor SS and Subramaniam S (2000) Domain architecture of a Caenorhabditis elegans AKAP suggests a novel AKAP function. FEBS Lett. 8: 107-111

49. Maier D, Nagel AC, Johannes B and Preiss A (1999) Subcellular localization of Hairless protein shows a major focus of activity within the nucleus. Mech. Dev. 89: 195-199

50. Ramos RG, Grimwade BG, Wharton KA, Scottgale TN and Artavanis-Tsakonas S (1989) Physical and functional definition of the Drosophila Notch locus by $P$ element transformation. Genetics 123: 337-348

51. Peterson C, Carney GE, Taylor BJ and White K (2002) reaper is required for neuroblast apoptosis during Drosophila development. Development 129: 1467-1476

52. Brand AH and Perrimon N (1993) Targeted gene expression as a means of altering cell fates and generating dominant phenotypes. Development 118 401-415

53. Brunner E, Ducker K, Oellers N, Hafen E, Scholz H and Klämbt C (1994) The ETS domain protein pointed-P2 is a target of MAP kinase in the sevenless signal transduction pathway. Nature 370: 386-389

54. Newsome TP, Asling B and Dickson BJ (2000) Analysis of Drosophila photoreceptor axon guidance in eye-specific mosaics. Development 127: 851-860

55. O'Keefe L, Dougan ST, Gabay L, Raz E, Shilo BZ and DiNardo S (1997) Spitz and Wingless, emanating from distinct borders, cooperate to establish cell fate across the Engrailed domain in the Drosophila epidermis. Development 124: 4837-4845

56. Tang CY and Sun $\mathrm{H}$ (2002) Use of mini-white as a reporter gene to screen for Gal4 insertions with spatially restricted expression pattern in the developing eye in Drosophila. Genesis 34: 39-45 